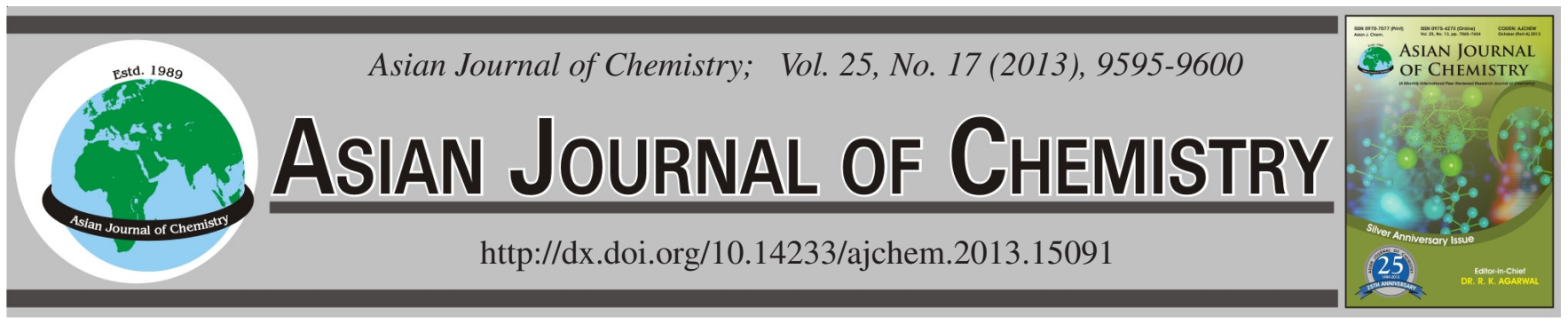

\title{
Synthesis of Novel Diarylpyrrole-2-carbaldehydes by Ring Transformations
}

\author{
Samina Aslam ${ }^{1}$, Areesha Nazeer ${ }^{2}$, Muhammad Naeem Khan ${ }^{3, *}$, \\ Najma Parveen ${ }^{4}$, Misbahul Ain Khan ${ }^{1}$ and Munawar Ali Munawar ${ }^{2}$
}

${ }^{1}$ Department of Chemistry, The Islamia University of Bahawalpur, Bahawalpur, Pakistan
${ }^{2}$ Institute of Chemistry, University of the Punjab, Quaid-i-Azam Campus, Lahore, Pakistan
${ }^{3}$ Applied Chemistry Research Centre, PCSIR Laboratories Complex, Lahore, Pakistan
${ }^{4}$ Department of Chemistry, The Government Sadiq College Women University, Bahawalpur, Pakistan

*Corresponding author: E-mail: changwani_1@yahoo.com

(Received: 4 January 2013;

Accepted: 4 October 2013)

AJC-14235

Various diarylpyrrole-2-carbaldehydes were prepared by ring transformation of arylfuran-2-carbaldehydes with anilines in the presence of an acid. The synthesized compounds were characterized through elemental analysis and spectroscopic techniques (FTIR, ${ }^{1} \mathrm{H}$ NMR, ${ }^{13} \mathrm{C}$ NMR and mass spectra).

Key Words: Diarylpyrrole aldehydes, Ring transformation, Arylfuran-2-carbaldehydes, Meerwein arylation.

\section{INTRODUCTION}

Pyrrole is one of the most important heterocyclic compounds, having increasingly importance in medicinal chemistry and organic synthesis ${ }^{1-5}$. Some of the recently isolated pyrrolecontaining marine natural products have been found to exhibit considerable cytotoxicity and function as multi drug resistant (MDR) reversal agents. Many of these biologically active compounds have emerged as chemotherapeutic agents ${ }^{6}$. Due to these multiple uses and varieties of biological activities, the synthesis of this ring system has been subject of intense investigation. Several new synthetic methods and variations of classical ones reported recently either give new types of pyrroles or result in their better yields. The classical methods of constructing pyrrole ring system include mainly conjugate addition reactions ${ }^{7}$, transition metal-mediated reactions ${ }^{8}$, reductive couplings ${ }^{9}$, aza-Wittig reactions ${ }^{10}$ and other multistep operations ${ }^{11}$ have all been performed for the synthesis of pyrroles. Despite these huge developments, the Paal-Knorr ${ }^{12}$ reaction is still considered to be the most attractive method for the synthesis of pyrroles.

Since various vicinal diarylazoles/heterocycles are known to be cycloxygenase (COX) inhibitors and some have been in clinical use e.g., celecoxib (1) and valdecoxib (2) $)^{13,14}$. These have come under criticism because of their adverse side effects $^{15,16}$, so there is a continuous effort in quest for finding better agents and diarylpyrroles seem to be drawing much attention of heterocyclic chemists in this regard.<smiles>Cc1ccc(-c2cc(C(F)(F)F)nn2-c2ccc(S(N)(=O)=O)cc2)cc1</smiles>

(1)<smiles>Cc1onc(-c2ccccc2)c1-c1ccc(S(N)(=O)=O)cc1</smiles>

(2)
Synthesis of 4,5-diarylpyrroles and their antiinflammatory activity has been reported by Wilkerson et al. ${ }^{17}$. Some 1,5-diarylpyrrole- $1 H$-3-acetic acids (3), esters and 3-alkoxyethyl ethers have been synthesized as potent COX-2 inhibitors $^{18-20}$. Interestingly these authors have also reported other 1,5-diarylpyrroles as potential antimycobacterial agents ${ }^{21,22}$.<smiles>[R]OS(=O)(=O)c1ccc(-c2cc(CC(=O)O)c(C)n2-c2ccc([R])cc2)cc1</smiles>

(3)

Various 1,5-diarylpyrroles were reported by Hall et al. ${ }^{23,24}$ as EP1 receptor antagonists. Most of these experimental 1,5- 
diarylpyrroles were prepared from appropriately substituted 1,4-diketones by the Paal-Knorr method ${ }^{12}$. 2,5-Dialkoxytetrahydrofurans are "masked" or cyclic acetals of 1,4-dialdehydes which either under acid catalysis ${ }^{25}$ or under microwave irradiation in the presence of iodine ${ }^{26}$ react with aromatic amines affording $\mathrm{N}$-arylpyrroles. A ring transformation of furan-2carbaldehyde to 1-arylpyrrole-2-carbaldehyde takes place in the presence of an arylamine and an acid catalyst ${ }^{27,28}$. This general reaction had provided us $\mathrm{N}$-arylpyrrole-2-carbaldehydes in good yields and has encouraged us to attempt these ring transformations with 5-arylfuran-2-carbaldehydes. The reactions were successful and here we would like to present our findings. It is to be noted that the resultant products carrying a carbaldehyde function opens up various possibilities of preparation of diverse derivatives of 1,5-diarylpyrroles.

\section{EXPERIMENTAL}

All reagents and solvents were used as obtained from the supplier or recrystallized or redistilled as were found necessary. Thin layer chromatography was performed using aluminium sheets (Merck) coated with silica gel $60 \mathrm{~F}_{254}$. IR spectra were recorded by using an IR Perkin-Elmer spectrum 1 FTIR spectrophotometer and peaks are reported max (neat) $/ \mathrm{cm}^{-1}$ which refer to the min wave numbers. Proton magnetic resonance spectra were recorded in deuteriochloroform with Bruker AM 300 spectrometers (Rheinstetten-Forchheim, Germany) operating at $300 \mathrm{MHz}$, respectively. The ${ }^{13} \mathrm{C}$ NMR spectra were recorded in Deuteriochloroform with Bruker AM 100 spectrometer operating at $100 \mathrm{MHz}$. Tetramethylsilane was used as an internal standard. Elemental analysis for $\mathrm{C}, \mathrm{H}$ and $\mathrm{N}$ were recorded with Perkin-Elmer 2400 Series II CHN Analyzer. Melting points were recorded on a GallenKamp apparatus and are uncorrected.

General procedure for the synthesis of 5-arylfuran-2carbaldehydes: Substituted aniline $(0.01 \mathrm{~mol})$ is dissolved in a mixture of conc. hydrochloric acid and $20 \mathrm{~mL}$ of water under stirring and cooled in an ice bath at $-5^{\circ} \mathrm{C}$. A solution of sodium nitrite ( $2 \mathrm{~g}$ in $10 \mathrm{~mL}$ of water) is added portion wise, keeping the temperature below $7-8{ }^{\circ} \mathrm{C}$. The reaction mixture is left for $1 \mathrm{~h}$ for the completion of diazotization, filtered with the help of glass wool (in case there is any turbidity observed). The filtered diazonium solution is added drop wise to a solution of furfural $(2 \mathrm{~mL}$ in $10 \mathrm{~mL}$ of acetone and water) followed by a solution of copper chloride ( $2 \mathrm{~g}$ in $10 \mathrm{~mL}$ of water). The temperature is raised to $30{ }^{\circ} \mathrm{C}$ by heating (if necessary) and stirred for 4-6 h then left for $24 \mathrm{~h}$ at room temperature. Precipitates obtained are filtered, dried and recrystallized from ethanol.

Following 5-arylfuran-2-carbaldehydes are prepared in this manner:

5-(4'-Nitrophenyl)furan-2-carbaldehyde (4): Yellow crystals, m.p. $196{ }^{\circ} \mathrm{C}(\mathrm{EtOH})$ (m.p. $192{ }^{\circ} \mathrm{C}(\mathrm{EtOH})^{33}$.

5-(4'-Chlorophenyl)furan-2-carbaldehyde (5): Light yellow crystals, m.p. $118^{\circ} \mathrm{C}(\mathrm{EtOH})\left(\right.$ m.p. $118^{\circ} \mathrm{C}(\mathrm{EtOH})^{33}$.

5-(4'-Bromophenyl)furan-2-carbaldehyde (6): Off white crystals, m.p. $150^{\circ} \mathrm{C}(\mathrm{EtOH})\left(\right.$ m.p. $150^{\circ} \mathrm{C}(\mathrm{EtOH})^{33}$.

4-(5'-Formylfuran-2'-yl)benzoic acid (7): Light brown crystals, m.p. $296^{\circ} \mathrm{C}(\mathrm{EtOH})$. FTIR $\left(\mathrm{KBr}, \nu_{\max }, \mathrm{cm}^{-1}\right): 2360.14$ (aromatic ring) 1672.39 (C=O acid) 2968.44 (-OH acid)
$1610.63\left(\mathrm{C}=\mathrm{O}\right.$ aldehyde) ${ }^{1} \mathrm{H}$ NMR spectrum $\left(\mathrm{CDCl}_{3}, 300\right.$ $\mathrm{MHz}), \delta$ ppm (J, Hz): 9.567 (1H, s, Ar-Cㅌㅇ); 7.797 (1H, d, $J=6.40, \operatorname{Ar}-\underline{\mathrm{H}}), 7.877(1 \mathrm{H}, \mathrm{d}, J=6.00, \mathrm{Ar}-\underline{\mathrm{H}}), 7.288(1 \mathrm{H}, \mathrm{d}$, $J=6.30, \mathrm{Ar}-\underline{\mathrm{H}})), 8.021(1 \mathrm{H}, \mathrm{d}, J=6.50, \mathrm{Ar}-\underline{\mathrm{H}}), 6.893(1 \mathrm{H}, \mathrm{d}$, $J=2.40$, furyl proton), $7.238(1 \mathrm{H}, \mathrm{d}, J=2.40$, furyl proton) ${ }^{13} \mathrm{C}$ NMR spectrum $\left(\mathrm{CDCl}_{3}, 75 \mathrm{MHz}\right) \delta \mathrm{ppm}: 178.51(\mathrm{Ar}-$

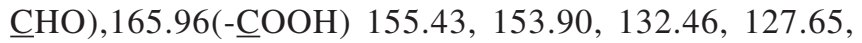
123.87, 122.43, 122.09, 117.78, 109.89 (Ar-C) mass spectrum (EI, $70 \mathrm{eV},\left(\mathrm{I}_{\text {rel }} \%\right): 216\left[\mathrm{M}^{+}\right](100), 199$ [M+- OH] (11) $m / z 170\left[\mathrm{M}^{+}-\mathrm{COOH}\right](7), m / z 76[\mathrm{Ph}](4)$, found (\%) C 66.71; $\mathrm{H}$ 3.76; $\mathrm{C}_{12} \mathrm{H}_{8} \mathrm{O}_{4}$ (216), calcd. (\%) : C 66.66; H 3.70.

General method for the ring transformation reaction of 5-arylfuran-2-carbaldehydes: Equimolar quantities (0.01 mole) of a 5-arylfuran-2-carbaldehyde and aniline are refluxed in $20 \mathrm{~mL}$ ethanol for $6 \mathrm{~h}$ in the presence of conc. hydrochloric acid $(0.5 \mathrm{~mL})$ as a catalyst and then poured the reaction mixture over crushed ice, the precipitates are filtered, dried and recrystallized from ethanol.

5-(4'-Nitrophenyl)-1-phenyl-1H-pyrrole-2-carbaldehyde (8): Reddish crystals, m.p. $102{ }^{\circ} \mathrm{C}(\mathrm{EtOH})$ FTIR KBr, $v_{\max }$, $\mathrm{cm}^{-1}$ ): 1599.16 (C=O aldehyde), 1538.50 and 1333.39 (asym and sym $\left.-\mathrm{NO}_{2}\right),{ }^{1} \mathrm{H}$ NMR spectrum $\left(\mathrm{CDCl}_{3}, 300 \mathrm{MHz}\right), \delta \mathrm{ppm}$ (J, Hz): 9.41 (s, 1H, CHO), 7.939-7.008 (m, 9H, Ar-H), 7.001 (d, $1 \mathrm{H}$, pyrrole-H $J=3.10 \mathrm{~Hz},), 6.678(\mathrm{~d}, 1 \mathrm{H}$, pyrrole-H, $J=$ $3.10 \mathrm{~Hz}){ }^{13} \mathrm{C}$ NMR spectrum $\left(\mathrm{CDCl}_{3}, 75 \mathrm{MHz}\right) \delta$ ppm: 175.65 $(\mathrm{C}=\mathrm{O}$ aldehyde $), 145.08,131.93,129.75,129.54,124.27$, 123.66, 123.56, 115.46, 115.16, 110.87, 109.89 (Ar-C) mass spectrum (EI, $70 \mathrm{eV},\left(\mathrm{I}_{\text {rel }} \%\right): \mathrm{m} / z 293\left[\mathrm{M}^{+}\right](20), 263\left[\mathrm{M}^{+}-\mathrm{CHO}\right]$ (10), 93 [ $\left.\mathrm{M}^{+}-\mathrm{PhNO}_{2}-\mathrm{Ph}\right]$ (13) found (\%) C 69.54; $\mathrm{H} 4.22 ; \mathrm{N}$ $9.89 \mathrm{C}_{17} \mathrm{H}_{12} \mathrm{~N}_{2} \mathrm{O}_{3}$ (293) calcd. (\%) C 69.86; H 4.10; N 9.59.

5-(4'-Nitrophenyl)-1-(4"-nitrophenyl)-1H-pyrrole-2carbaldehyde (9): Dark red crystals, m.p. $124^{\circ} \mathrm{C}(\mathrm{EtOH})$ FTIR $\left.\mathrm{KBr}, v_{\max }, \mathrm{cm}^{-1}\right): 2362.53$ (aromatic ring) $1628.46(\mathrm{C}=\mathrm{O}$ aldehyde), 1587.22 and 1296.40 (asym and sym $-\mathrm{NO}_{2}$ ) ${ }^{1} \mathrm{H}$ NMR spectrum $\left(\mathrm{CDCl}_{3}, 300 \mathrm{MHz}\right), \delta \mathrm{ppm}(\mathrm{J}, \mathrm{Hz}): 9.71(\mathrm{~s}, 1 \mathrm{H}, \mathrm{CHO})$, 8.306-7.026 (m, 8H, Ar-H), 7.017 (d, 1H, pyrrole-H $J=3.45$ $\mathrm{Hz}), 6.594(\mathrm{~d}, 1 \mathrm{H}$, pyrrole- $\mathrm{H}, J=3.45 \mathrm{~Hz}){ }^{13} \mathrm{C}$ NMR spectrum $\left(\mathrm{CDCl}_{3}, 75 \mathrm{MHz}\right) \delta \mathrm{ppm}: 177.54(\mathrm{C}=\mathrm{O}$ aldehyde),134.58, $126.33,125.78,125.57,125.21,124.43,123.86,123.63$, 122.63, 121.48, 113.41, 109.75 (Ar-C) Mass spectrum (EI, $70 \mathrm{eV},\left(\mathrm{I}_{\text {rel }} \%\right): \mathrm{m} / \mathrm{z} 337\left[\mathrm{M}^{+}\right]$(11), $215\left[\mathrm{M}^{+}-\mathrm{PhNO}_{2}\right](100), 187$ $\left[\mathrm{M}^{+}-\mathrm{PhNO}_{2}-\mathrm{CHO}\right]$ (47) found (\%) C 60.34; H 3.05; N 12.26 $\mathrm{C}_{17} \mathrm{H}_{11} \mathrm{~N}_{3} \mathrm{O}_{5}$ (337) calcd. (\%) C 60.53; H 3.26; N 12.46.

5-(4'-Nitrophenyl)-1-(4"'-chlorophenyl)-1H-pyrrole-2carbaldehyde (10): Brown crystals, m.p. $124^{\circ} \mathrm{C}(\mathrm{EtOH})$ FTIR $\mathrm{KBr}, v_{\max }, \mathrm{cm}^{-1}$ ): 2360.42 (aromatic ring) $1684.99 \mathrm{~cm}^{-1}(\mathrm{C}=\mathrm{O}$ aldehyde), 1042.43 (C-Cl bond), 1600.22 and 1358.09 (asym - $\left.\mathrm{NO}_{2}\right),{ }^{1} \mathrm{H}$ NMR spectrum $\left(\mathrm{CDCl}_{3}, 300 \mathrm{MHz}\right), \delta \mathrm{ppm}(\mathrm{J}, \mathrm{Hz})$ : 9.718 (s, 1H, CHO), 8.312-7.341 (m, 8H, Ar-H), 7.029 (d, $1 \mathrm{H}$, pyrrole-H $J=2.80 \mathrm{~Hz}), 7.017(\mathrm{~d}, 1 \mathrm{H}$, pyrrole-H, $J=2.80$ $\mathrm{Hz})^{13} \mathrm{C} \mathrm{NMR}$ spectrum $\left(\mathrm{CDCl}_{3}, 75 \mathrm{MHz}\right) \delta \mathrm{ppm}: 177.55(\mathrm{C}=\mathrm{O}$ aldehyde), 142.55, 131.78, 129.48, 125.76, 125.23, 124.42, 122.68, 122.31, 110.62 (Ar-C) mass spectrum (EI, $70 \mathrm{eV}$, $\left.\left(\mathrm{I}_{\text {rel }} \%\right): m / z, 28\left[\mathrm{M}^{+}+2\right], 326\left[\mathrm{M}^{+}\right](7.1), 215 \mathrm{M}^{+}-\mathrm{PhCl}\right](100)$ Found (\%) C 62.32; H 3.13; N $8.44 \mathrm{C}_{17} \mathrm{H}_{11} \mathrm{ClN}_{2} \mathrm{O}_{3}$ (326) calcd. (\%) C 62.57 ; H 3.37 ; N 8.58.

5-(4'-Nitrophenyl)-1-(4"'-bromophenyl)-1H-pyrrole-2carbaldehyde (11): Yellow crystals. m.p. $232^{\circ} \mathrm{C}(\mathrm{EtOH})$. FTIR $\left.\mathrm{KBr}, \mathrm{v}_{\max }, \mathrm{cm}^{-1}\right): 1655.84(\mathrm{C}=\mathrm{O}$ aldehyde $), 1594.41$ and 
$1361.51\left(-\mathrm{NO}_{2}\right), 2365.43$ (aromatic ring), $1037.49 \mathrm{~cm}^{-1}(\mathrm{C}-\mathrm{Br}$ bond) ${ }^{1} \mathrm{H}$ NMR spectrum $\left(\mathrm{CDCl}_{3}, 300 \mathrm{MHz}\right), \delta \mathrm{ppm}(J, \mathrm{~Hz})$ : 9.636 (s, 1H, CHO), 8.360-7.618 (m, 8H, Ar-H), 7.019 (d, $1 \mathrm{H}$, pyrrole-H, $J=3.15 \mathrm{~Hz}$ ), $6.655(\mathrm{~d}, 1 \mathrm{H}$, pyrrole-H, $J=$ $3.15 \mathrm{~Hz}){ }^{13} \mathrm{C}$ NMR spectrum $\left(\mathrm{CDCl}_{3}, 75 \mathrm{MHz}\right) \delta \mathrm{ppm}: 177.45$ ( $\mathrm{C}=\mathrm{O}$ aldehyde $), 142.66,132.34,132.23,131.83,131.60$, $131.11,130.93,126.71,125.23,113.91,108.06$ (Ar-C) mass spectrum (EI, $70 \mathrm{eV},\left(\mathrm{I}_{\text {rel }} \%\right): \mathrm{m} / z 372\left[\mathrm{M}^{+}+2\right](100), 370\left[\mathrm{M}^{+}\right]$ (91) found (\%) C 54.98; $\mathrm{H} 2.76 ; \mathrm{N} 7.44 \mathrm{C}_{17} \mathrm{H}_{11} \mathrm{BrN}_{2} \mathrm{O}_{3}(370)$ calcd. (\%) C 55.13; H 2.97; N 7.56.

4-(5'-Formyl-1-(4'-nitrophenyl)-1H-pyrrol-1-yl)benzoic acid (12): Brown crystals, m.p. $150{ }^{\circ} \mathrm{C}(\mathrm{EtOH})$ FTIR (KBr, $\left.v_{\max }, \mathrm{cm}^{-1}\right)$ : $\mathrm{H} 2363.72$ (aromatic ring), 1715.12 ( $\mathrm{C}=\mathrm{O}$ acid), 1686.17 ( $\mathrm{C}=\mathrm{O}$ aldehyde), 1599.94 and 1341.94 (Asym and sym $\left.-\mathrm{NO}_{2}\right), 3114.28$ (-OH acid) ${ }^{1} \mathrm{H}$ NMR spectrum $\left(\mathrm{CDCl}_{3}\right.$, $300 \mathrm{MHz}$ ), $\delta$ ppm (J, Hz): 9.721 (s, 1H, CHO), 8.317-7.342 (m, 8H, Ar-H), 7.029 (d, 1H, pyrrole-H, $J=2.85 \mathrm{~Hz}$ ), 7.017 $(\mathrm{d}, 1 \mathrm{H}$, pyrrole- $\mathrm{H}, J=2.85 \mathrm{~Hz}),{ }^{13} \mathrm{C}$ NMR spectrum $\left(\mathrm{CDCl}_{3}\right.$, $75 \mathrm{MHz}) \delta$ ppm: 178.89 (C=O aldehyde),140.36, 131.58, $130.87,130.21,128.90,127.60,125.03,113.83,109.31$, 106.55 (Ar-C) Mass spectrum (EI, $70 \mathrm{eV},\left(\mathrm{I}_{\text {rel }} \%\right): \mathrm{m} / z, 336\left[\mathrm{M}^{+}\right]$ (78), $306\left[\mathrm{M}^{+}-\mathrm{CHO}\right](66), 289\left[\mathrm{M}^{+}-\mathrm{NO} 2\right]$ ( 32) found (\%) C 64.21; H 3.46; $\mathrm{N} 8.21 \mathrm{C}_{18} \mathrm{H}_{12} \mathrm{~N}_{2} \mathrm{O}_{5}$ (336) calcd. (\%)C 64.28; $\mathrm{H}$ 3.57 ; N 8.33.

5-(4'-Chlorophenyl)-1-phenyl-1H-pyrrole-2-carbaldehyde (13): Yellow crystals, m.p. $82^{\circ} \mathrm{C}(\mathrm{EtOH})$ FTIR $(\mathrm{KBr}$, $\left.v_{\max }, \mathrm{cm}^{-1}\right)$ : 1614.87 ( $\mathrm{C}=\mathrm{O}$ aldehyde), 1092.01 (C-Br bond)), ${ }^{1} \mathrm{H} \mathrm{NMR}$ spectrum $\left(\mathrm{CDCl}_{3}, 300 \mathrm{MHz}\right), \delta \mathrm{ppm}(\mathrm{J}, \mathrm{Hz}): 9.610(\mathrm{~s}$, 1H, CHO), 7.707-7.189 (m, 8H, Ar-H), 7.033 (d, 1H, pyrrole$\mathrm{H}, J=3.50 \mathrm{~Hz}), 6.514(\mathrm{~d}, 1 \mathrm{H}$, pyrrole-H, $J=3.50 \mathrm{~Hz}){ }^{13} \mathrm{C}$ NMR spectrum $\left(\mathrm{CDCl}_{3}, 75 \mathrm{MHz}\right) \delta$ ppm: $178.90(\mathrm{C}=\mathrm{O}$ aldehyde), 144.87, 132.43, 129.74, 129.61, 128.70, 125.95, 125.64, 125.39, 124.73,123.13, 121.04, 110.08, 106.04(Ar-C) mass spectrum (EI, $70 \mathrm{eV},\left(\mathrm{I}_{\mathrm{rel}} \%\right): \mathrm{m} / z 284\left[\mathrm{M}^{+}+2\right], 282\left[\mathrm{M}^{+}\right](40)$, $254\left[\mathrm{M}^{+}-\mathrm{CHO}\right](5)$ found (\%) C 72.25; H 4.52; N 4.76 $\mathrm{C}_{17} \mathrm{H}_{12} \mathrm{ClNO}$ (281) calcd. (\%) C 72.59; H 4.27; N 4.98.

5-(4'-Chlorophenyl)-1-(4"'-nitrophenyl)-1H-pyrrole-2carbaldehyde (14): Light brown crystals m.p. $165^{\circ} \mathrm{C}(\mathrm{EtOH})$ FTIR $\left(\mathrm{KBr}, v_{\max }, \mathrm{cm}^{-1}\right): 1628.38(\mathrm{C}=\mathrm{O}$ aldehyde $), 1584.86$ and 1328.36 (asym and sym - $\mathrm{NO}_{2}$ ), 1094.51 (C-Cl bond), ${ }^{1} \mathrm{H}$ NMR spectrum $\left(\mathrm{CDCl}_{3}, 300 \mathrm{MHz}\right), \delta \mathrm{ppm}(\mathrm{J}, \mathrm{Hz}): 9.638(\mathrm{~s}, 1 \mathrm{H}$, CHO), 8.072-7.295 (m, 8H, Ar-H), 7.322 (d, 1H, pyrrole-H, $J=3.20 \mathrm{~Hz}), 6.821(\mathrm{~d}, 1 \mathrm{H}$, pyrrole- $\mathrm{H}, J=3.20 \mathrm{~Hz}){ }^{13} \mathrm{C} \mathrm{NMR}$ spectrum $\left(\mathrm{CDCl}_{3}, 75 \mathrm{MHz}\right) \delta \mathrm{ppm}: 177.22(\mathrm{C}=\mathrm{O}$ aldehyde $)$, $152.37,149.29,129.27,128.95,126.52,126.33,125.11$, $124.90,121.45,113.37,108.50,107.97$ (Ar-C) mass spectrum (EI, $70 \mathrm{eV},\left(\mathrm{I}_{\text {rel }} \%\right): \mathrm{m} / z, 328\left[\mathrm{M}^{+}+2\right], 326\left[\mathrm{M}^{+}\right](72), 205$ $\left[\mathrm{M}^{+}-\mathrm{PhNO}_{2}\right]$ (100) found (\%) C 62.13; H 3.11; N 8.64 $\mathrm{C}_{17} \mathrm{H}_{11} \mathrm{ClN}_{2} \mathrm{O}_{3}$ (326) calcd. (\%) C 62.57; H 3.37; N 8.58.

5-(4'-Chlorophenyl)-1-(4"-chlorophenyl) -1H-pyrrole2-carbaldehyde (15): Dark red crystals, m.p. $196^{\circ} \mathrm{C}(\mathrm{EtOH})$; FTIR $\left(\mathrm{KBr}, v_{\max }, \mathrm{cm}^{-1}\right): 1658.80(\mathrm{C}=\mathrm{O}$ aldehyde), 2563.01 (aromatic ring), 1092.17(C-Cl bond) ${ }^{1} \mathrm{H}$ NMR spectrum $\left(\mathrm{CDCl}_{3}, 300 \mathrm{MHz}\right), \delta \mathrm{ppm}(\mathrm{J}, \mathrm{Hz}): 9.639$ (s, 1H, CHO), 7.754$7.293(\mathrm{~m}, 8 \mathrm{H}, \mathrm{Ar}-\mathrm{H}), 7.220$ (d, 1H, pyrrole-H, $J=3.50 \mathrm{~Hz}$ ), $6.808(\mathrm{~d}, 1 \mathrm{H}$, pyrrole- $\mathrm{H}, J=3.50 \mathrm{~Hz}) .{ }^{13} \mathrm{C}$ NMR spectrum $\left(\mathrm{CDCl}_{3}, 75 \mathrm{MHz}\right) \delta \mathrm{ppm}: 177.21(\mathrm{C}=\mathrm{O}$ aldehyde), 135.70, $129.29,129.15,128.78,127.51,126.53,125.65,123.16$, 118.45, 108.61, 107.97 (Ar-C) mass spectrum (EI, $70 \mathrm{eV}$,
$\left(\mathrm{I}_{\mathrm{rel}} \%\right): \mathrm{m} / z, 317\left[\mathrm{M}^{+}+2\right], 315\left[\mathrm{M}^{+}\right](100), 204\left[\mathrm{M}^{+}-\mathrm{PhCl}\right](12)$, $111[\mathrm{PhCl}](45) 75[\mathrm{Ph}](27), 139$ [PhCl] (69), $280\left[\mathrm{M}^{+}-\mathrm{Cl}\right]$ (11) found (\%) C 64.31; $\mathrm{H} 3.18 ; \mathrm{N} 4.32$ ), $\mathrm{C}_{17} \mathrm{H}_{11} \mathrm{Cl}_{2} \mathrm{NO}$ (315) calcd. (\%) C 64.55; H 3.48; N 4.43

5-(4'-Chlorophenyl)-1-(4"'-bromophenyl) -1H-pyrrole2-carbaldehyde (16): Brown crystals, m.p. $98^{\circ} \mathrm{C}(\mathrm{EtOH})$ FTIR $\left(\mathrm{KBr}, v_{\max }, \mathrm{cm}^{-1}\right)$ : 1677.94 (C=O aldehyde), 2322.89 (aromatic ring), 1094.65 (C-Cl bond), 1040.37 (C-Br bond), ${ }^{1} \mathrm{H}$ NMR spectrum $\left(\mathrm{CDCl}_{3}, 300 \mathrm{MHz}\right), \delta \mathrm{ppm}(\mathrm{J}, \mathrm{Hz}): 9.636(\mathrm{~s}, 1 \mathrm{H}$, CHO), 7.750-7.292 (m, 8H, Ar-H), 6.818 (d, 1H, pyrrole-H, $J=7.50 \mathrm{~Hz}), 6.204$ (d, $1 \mathrm{H}$, pyrrole- $\mathrm{H}, J=2.80 \mathrm{~Hz}){ }^{13} \mathrm{C} \mathrm{NMR}$ spectrum $\left(\mathrm{CDCl}_{3}, 75 \mathrm{MHz}\right) \delta$ ppm: 177.18 (C=O aldehydes) $158.19,152.20,135.67,129.26,128.94,127.49,126.50$, 124.94, 123.27, 107.95 (Ar-C) mass spectrum (EI, $70 \mathrm{eV}$, (I $\mathrm{I}_{\text {rel }}$ $\%): m / z 361\left[\mathrm{M}^{+}+2\right](100), 359\left[\mathrm{M}^{+}\right](72), 111[\mathrm{PhCl}](43)$ found (\%) C 56.91; H 3.10; N 3.56 $\mathrm{C}_{17} \mathrm{H}_{11} \mathrm{BrClNO}$ (359) calcd. (\%) C $56.82 ;$ H 3.06 ; N 3.89.

4-(2"-Formyl-5-(4'-chlorophenyl)-1H-pyrrol-1-yl) benzoic acid (17): Light yellow crystals, m.p. $212^{\circ} \mathrm{C}(\mathrm{EtOH})$ FTIR $\left(\mathrm{KBr}, v_{\max }, \mathrm{cm}^{-1}\right)$ : 2341.29 (aromatic ring) $1717.11(\mathrm{C}=\mathrm{O}$ acid), 1659.90 ( $\mathrm{C}=\mathrm{O}$ aldehyde) 3115.21 (-OH acid), 1094.88 (C-Cl bond), ${ }^{1} \mathrm{H}$ NMR spectrum $\left(\mathrm{CDCl}_{3}, 300 \mathrm{MHz}\right), \delta \mathrm{ppm}(J$, Hz): 9.639 (s, 1H, CHO), 7.755-7.293 (m, 8H, Ar-H), 7.221 $(\mathrm{d}, 1 \mathrm{H}$, pyrrole-H, $J=3.20 \mathrm{~Hz}), 6.808(\mathrm{~d}, 1 \mathrm{H}$, pyrrole- $\mathrm{H}, J=$ $3.20 \mathrm{~Hz}), 11.559(\mathrm{COOH}) .{ }^{13} \mathrm{C}$ NMR spectrum $\left(\mathrm{CDCl}_{3}, 75\right.$ $\mathrm{MHz}) \delta$ ppm: 177.45 (C=O aldehyde), 147.59, 134.92, 133.56, 130.24, 129.63, 129.22, 125.05, 124.44, 123.12, 121.28, 110.72, 108.01 (Ar-C) mass spectrum (EI, $70 \mathrm{eV},\left(\mathrm{I}_{\text {rel }} \%\right): \mathrm{m} / \mathrm{z}$ $327\left[\mathrm{M}^{+}+2\right], 325\left[\mathrm{M}^{+}\right](53), 279\left[\mathrm{M}^{+}-\mathrm{COOH}\right](33), 217\left[\mathrm{M}^{+}-\right.$ $\mathrm{PhCOOH}$ ] (33) Found 5 C 66.08; H 3.59; N $3.98 \mathrm{C}_{18} \mathrm{H}_{12} \mathrm{ClNO}_{3}$ (325) calcd. (\%) C 66.46; H 3.69; N 4.30.

5-(4' - Bromophenyl) - 1 - phenyl-1H-pyrole-2 carbaldehyde (18): Red crystals, m.p. $122{ }^{\circ} \mathrm{C}(\mathrm{EtOH})$ FTIR $\left(\mathrm{KBr}, v_{\max }, \mathrm{cm}^{-1}\right): 2341.84$ (aromatic ring) $1661.86(\mathrm{C}=\mathrm{O}$ aldehyde) ${ }^{1} \mathrm{H}$ NMR spectrum $\left(\mathrm{CDCl}_{3}, 300 \mathrm{MHz}\right), \delta \mathrm{ppm}(J$, Hz): 9.635 (s, 1H, CHO), 8.282 (d, 2H, Ar-H), 7.680 (d, 2H, Ar-H, $J=4.50 \mathrm{~Hz}) 7.572$ (d, 1H, Ar-H, $J=4.50 \mathrm{~Hz}), 7.441$ (d, 1H, Ar-H), 7.299 (d, 1H, 7.220 (d, pyrrole-H, $J=2.75 \mathrm{~Hz}$ ), 6.827 (d, pyrrole- $\mathrm{H}, J=2.75 \mathrm{~Hz}){ }^{13} \mathrm{C}$ NMR spectrum $\left(\mathrm{CDCl}_{3}\right.$, $75 \mathrm{MHz}) \delta \mathrm{ppm}$ : 177.23 (C=O aldehyde), $166.39(\mathrm{COOH})$, $165.91(\mathrm{C}=\mathrm{O}$ ester $), 158.22,152.18,132.21,131.97,131.72$, 129.60, 127.89, 126.18, 125.09, 123.94, 123.34, 121.18, 108.04 (Ar-C) mass spectrum (EI, $70 \mathrm{eV},\left(\mathrm{I}_{\text {rel }}(\%)\right): \mathrm{m} / \mathrm{z} 327$ $\left[\mathrm{M}^{+}+2\right], 325\left[\mathrm{M}^{+}\right](12), 250\left[\mathrm{M}^{+}-\mathrm{Ph}\right](100)$ found $(\%) \mathrm{vC}$ 62.98; H 3.43; N 4.61 $\mathrm{C}_{17} \mathrm{H}_{12} \mathrm{BrNO}$ (325) calcd. (\%) C 62.76; H 3.69; N 4.30 .

5-(4'-Bromophenyl)-1-(4"-nitrophenyl)-1H-pyrrole-2carbaldehyde (19): Brown crystals, m.p. $128^{\circ} \mathrm{C}(\mathrm{EtOH})$ FTIR $\left(\mathrm{KBr}, \mathrm{v}_{\max }, \mathrm{cm}^{-1}\right): 2363.80$ (aromatic ring) $1628.71(\mathrm{C}=\mathrm{O}$ aldehyde), 1586.45 and 1298.23 (asym and sym $-\mathrm{NO}_{2}$ ), 1033.65 (C-Br bond), ${ }^{1} \mathrm{H}$ NMR spectrum $\left(\mathrm{CDCl}_{3}, 300 \mathrm{MHz}\right), \delta \mathrm{ppm}$ (J, Hz) : 9.642 (s, 1H, CHO), 7.564 (d, 2H, Ar-H), 7.521 (d, 2H, Ar-H), 7.492 (d, 1H, Ar-H), 7.304 (d, 1H, Ar-H,) 6.721 (d, pyrrole-H, $J=3.50 \mathrm{~Hz}), 6.622(\mathrm{~d}$, pyrrole- $\mathrm{H}, J=3.50 \mathrm{~Hz}){ }^{13} \mathrm{C}$ NMR spectrum $\left(\mathrm{CDCl}_{3}, 75 \mathrm{MHz}\right) \delta \mathrm{ppm}: 177.47(\mathrm{C}=\mathrm{O}$ aldehyde), 149.38, 130.24, 126.33, 125.12, 125.06, 124.70, 123.06, 121.47, 109.36 (Ar-C) mass spectrum (EI, $70 \mathrm{eV}$, ( $\left.\mathrm{I}_{\mathrm{rel}}(\%)\right)$ : $\mathrm{m} / \mathrm{z} 372\left[\mathrm{M}^{+}+2\right], 370\left[\mathrm{M}^{+}\right]$(100), $342\left[\mathrm{M}^{+}-\mathrm{CHO}\right]$ (50), 325 $\left[\mathrm{M}^{+}-\mathrm{NO}_{2}\right](25), 245\left[\mathrm{M}^{+}-\mathrm{PhNO}_{2}\right]$ (22), $217\left[\mathrm{M}^{+}-\mathrm{PhBr}\right]$ (11) 
found (\%) C 54.90; $\mathrm{H} 2.70 ; \mathrm{N} 7.21 \mathrm{C}_{17} \mathrm{H}_{11} \mathrm{BrN}_{2} \mathrm{O}_{3}$ (370) calcd. (\%) C 55.13; H 2.97; N 7.56.

5-(4'-Bromophenyl)-1-(4"'-chlorophenyl) -1H-pyrrole2-carbaldehyde (20): Dark brown crystals, m.p. $182{ }^{\circ} \mathrm{C}$ $(\mathrm{EtOH})$ FTIR $\left(\mathrm{KBr}, v_{\max }, \mathrm{cm}^{-1}\right): 2360.76$ (aromatic ring) 1660.03 ( $\mathrm{C}=\mathrm{O}$ aldehyde) 1073.87 (C-Cl bond), 1041.48 (C-Br bond), ${ }^{1} \mathrm{H}$ NMR spectrum $\left(\mathrm{CDCl}_{3}, 300 \mathrm{MHz}\right), \delta \mathrm{ppm}$ (J, Hz): 9.639 (s, 1H, CHO), 7.687 (d, 2H, Ar-H), 7.651 (d, 2H, Ar-H) 7.578 (d, 1H, Ar-H), 7.304 (d, 1H, Ar-H,), 6.833 (d, pyrrole-H, $J=3.15 \mathrm{~Hz}$ ), 6.821 (d, pyrrole-H, $J=3.15 \mathrm{~Hz}$ ). ${ }^{13} \mathrm{C}$ NMR spectrum $\left(\mathrm{CDCl}_{3}, 75 \mathrm{MHz}\right) \delta \mathrm{ppm}: 177.25(\mathrm{C}=\mathrm{O}$ aldehyde),142.33, 133.97, 132.22, 126.71, 125.41, 123.56, 121.09, 108.06 (Ar-C) mass spectrum (EI, $70 \mathrm{eV},\left(\mathrm{I}_{\text {rel }} \%\right)$ : $\mathrm{m} / \mathrm{z} 361\left[\mathrm{M}^{+}+2\right]$ (100), $359\left[\mathrm{M}^{+}\right]$(75), $326\left[\mathrm{M}^{+}+2-\mathrm{Cl}\right]$ (5), 280 $\left[\mathrm{M}^{+}-\mathrm{Br}\right](5.5), 155[\mathrm{PhBr}](5.3), 250\left[\mathrm{M}^{+}+2-\mathrm{PhCl}\right]$ (40) Found (\%) C 56.97; H 2.91; N $3.65 \mathrm{C}_{17} \mathrm{H}_{11} \mathrm{BrClNO}$ (359) calcd. (\%) C $56.82 ;$ H 3.06; N 3.90.

5-(4'-Bromophenyl)-1-(4"'-bromophenyl) -1H-pyrrole2-carbaldehyde (21): Reddish crystals m.p. $142{ }^{\circ} \mathrm{C}(\mathrm{EtOH})$ FTIR (KBr, $\left.v_{\max }, \mathrm{cm}^{-1}\right)$ : 2341.15 (aromatic ring) $1679.42(\mathrm{C}=\mathrm{O}$ aldehyde), 1041.68 (C-Br bond), ${ }^{1} \mathrm{H}$ NMR spectrum $\left(\mathrm{CDCl}_{3}\right.$, $300 \mathrm{MHz}), \delta \mathrm{ppm}(\mathrm{J}, \mathrm{Hz}): 9.638(\mathrm{~s}, 1 \mathrm{H}, \mathrm{CHO}), 7.686(\mathrm{~d}, 2 \mathrm{H}$, Ar-H), 7.658 (d, 2H, Ar-H,), 7.577 (d, 1H, Ar-H, J = 7.80 Hz, $J=8.90 \mathrm{~Hz}$ ), $7.303(\mathrm{~d}, 1 \mathrm{H}, \mathrm{Ar}-\mathrm{H}, J=9.80 \mathrm{~Hz}, J=8.60 \mathrm{~Hz})$, 6.832 (d, pyrrole-H, $J=2.90 \mathrm{~Hz}), 6.820(\mathrm{~d}$, pyrrole- $\mathrm{H}, J=$ $2.90 \mathrm{~Hz}){ }^{13} \mathrm{C}$ NMR spectrum $\left(\mathrm{CDCl}_{3}, 75 \mathrm{MHz}\right) \delta \mathrm{ppm}: 177.24$ $(\mathrm{C}=\mathrm{O}$ aldehyde $), 158.24,142.89,132.24,127.93,126.72$, 123.96, 108.07 (Ar-C) mass spectrum (EI, $70 \mathrm{eV},\left(\mathrm{I}_{\mathrm{rel}} \%\right): \mathrm{m} / \mathrm{z}$ $405\left[\mathrm{M}^{+} \mathrm{l}\right]$ (57), $326\left[\mathrm{M}^{+}-\mathrm{Br}\right]$ (4), $250\left[\mathrm{M}^{+}-\mathrm{PhBr}\right]$ (100), 155 [PhBr] (11), 222 [M+-PhBr-CHO] (11) found (\%) C 50.34; H $2.65 ; \mathrm{N} 3.21 \mathrm{C}_{17} \mathrm{H}_{11} \mathrm{Br}_{2} \mathrm{NO}$ (403) calcd. (\%) C 50.62; H 2.73; N 3.47.

4-(2"-Formyl-5-(4'-bromophenyl)-1H-pyrrol-1-yl) benzoic acid (22): Yellow crystals, m.p. $192^{\circ} \mathrm{C}(\mathrm{EtOH})$ FTIR $\left(\mathrm{KBr}, v_{\max }, \mathrm{cm}^{-1}\right): 2363.82$ (aromatic ring) $1681.83(\mathrm{C}=\mathrm{O}$ aldehyde), 1041.51 (C-Br bond). NMR spectrum $\left(\mathrm{CDCl}_{3}, 300\right.$ $\mathrm{MHz}$ ), $\delta$ ppm (J, Hz): 9.640 (s, 1H, CHO), 7.893 (d, 2H, ArH), 7.688 (d, 2H, Ar-H, $J=8.60 \mathrm{~Hz}), 7.578$ (d, 1H, Ar-H, $J=$ $8.6 \mathrm{~Hz}$ ), 7.304 (d, 1H, Ar-H, $J=8.50 \mathrm{~Hz}$ ), 6.833 (d, pyrrole$\mathrm{H}, J=3.30 \mathrm{~Hz}) 6.652$ (d, pyrrole-H, $J=3.30 \mathrm{~Hz}$ ), 11.583 $(\mathrm{COOH}){ }^{13} \mathrm{C}$ NMR spectrum $\left(\mathrm{CDCl}_{3}, 75 \mathrm{MHz}\right) \delta \mathrm{ppm}: 177.29$ $(\mathrm{C}=\mathrm{O}$ aldehyde $), 158.24(\mathrm{COOH}) 148.72,132.39,132.23$, $132.08,131.90,127.92,126.71,125.87,125.23,123.96$, 120.96, 111.89, 108.05 (Ar-C) mass spectrum (EI, $70 \mathrm{eV}$, (I $\mathrm{I}_{\text {rel }}$ $\%): m / z 371\left[\mathrm{M}^{+}+2\right], 369\left[\mathrm{M}^{+}\right](49), 290\left[\mathrm{M}^{+}-\mathrm{Br}\right](6), 214\left[\mathrm{M}^{+}-\right.$ $\mathrm{PhBr}]$ (4), 155 [PhBr] (8) Found (\%) C 61.05; H 3.56; N 6.71 $\mathrm{C}_{18} \mathrm{H}_{12} \mathrm{BrNO}_{3}$ (369) calcd. (\%)C 60.81; H 3.22; N 6.45

4-(5'-Formyl-1-phenyl-1H-pyrrol-2-yl) benzoic acid (23): Yellow crystals, m.p. $136{ }^{\circ} \mathrm{C}(\mathrm{EtOH})$ FTIR $\left(\mathrm{KBr}, \mathrm{v}_{\max }\right.$, $\mathrm{cm}^{-1}$ ): 2341.82(aromatic ring), 1690.43 (C=O acid), 1678.61 $\left(\mathrm{C}=\mathrm{O}\right.$ aldehyde), ${ }^{1} \mathrm{H}$ NMR spectrum $\left(\mathrm{CDCl}_{3}, 300 \mathrm{MHz}\right), \delta$ $\operatorname{ppm}(\mathrm{J}, \mathrm{Hz})$ : 9.637 (s, 1H, CHO), 8.030-7.420 (m, 8H, Ar-H), $6.978(\mathrm{~d}, 1 \mathrm{H}$, pyrrole-H, $J=3.15 \mathrm{~Hz}), 6.658$ (d, 1H, pyrrole$\mathrm{H}, J=3.15 \mathrm{~Hz}){ }^{13} \mathrm{C} \mathrm{NMR}$ spectrum $\left(\mathrm{CDCl}_{3}, 75 \mathrm{MHz}\right) \delta \mathrm{ppm}$ : $178.35(\mathrm{C}=\mathrm{O}$ aldehyde $) 155.67,131.58,130.26,130.21$, 128.91, 125.07, 109.34 (Ar-C) Mass spectrum (EI, $70 \mathrm{eV}$, $\left(\mathrm{I}_{\text {rel }} \%\right): m / z 290\left[\mathrm{M}^{+}\right](7), 77[\mathrm{Ph}](38), 93$ [PyrroleCHO] (100) found (\%) $\mathrm{C} 74.01 ; \mathrm{H} 4.35 ; \mathrm{N} 5.05 \mathrm{C}_{18} \mathrm{H}_{13} \mathrm{NO}_{3}$ (291) calcd. C 74.22; H 4.46; N 4.81 .
4-(5'-Formyl-1-(4"-nitrophenyl)-1H-pyrrol-2-yl) benzoic acid (24): Red colour crystals, m.p. $182^{\circ} \mathrm{C}$, (EtOH) FTIR ( $\left.\mathrm{KBr}, v_{\max }, \mathrm{cm}^{-1}\right): 1679.78(\mathrm{C}=\mathrm{O}$ acid $), 1627.13(\mathrm{C}=\mathrm{O}$ aldehyde) 1586.60 and 1331.93 (asym and sym $-\mathrm{NO}_{2}$ ), 3365.23 (-OH acid), ${ }^{1} \mathrm{H}$ NMR spectrum $\left(\mathrm{CDCl}_{3}, 300 \mathrm{MHz}\right), \delta$ ppm $(J$, Hz): 9.692 (s, 1H, CHO), 8.069-8.039 (m, 8H, Ar-H), 6.620 (d, $1 \mathrm{H}$, pyrrole-H, $J=2.85 \mathrm{~Hz}), 6.590$ (d, $1 \mathrm{H}$, pyrrole- $\mathrm{H}, J=$ $2.85 \mathrm{~Hz}),{ }^{13} \mathrm{C}$ NMR spectrum $\left(\mathrm{CDCl}_{3}, 75 \mathrm{MHz}\right) \delta \mathrm{ppm}: 177.45$ ( $\mathrm{C}=\mathrm{O}$ aldehyde), 166.78, 147.96, 142.63, 132.86, 130.82, 130.26, 130.22, 125.07, 125.03, 122.91, 109.35 (Ar-C) mass spectrum (EI, $70 \mathrm{eV},\left(\mathrm{I}_{\text {rel }} \%\right): \mathrm{m} / z 336\left[\mathrm{M}^{+}\right](100), 306\left[\mathrm{M}^{+}-\right.$ $\mathrm{CHO}]$ (26), $289\left[\mathrm{M}^{+}-\mathrm{NO}_{2}\right]$ (19), $216\left[\mathrm{M}^{+}-\mathrm{PhCOOH}\right]$ (6) found (\%) $\mathrm{C} 64.34 ; \mathrm{H} 3.36 ; \mathrm{N} 8.57 \mathrm{C}_{18} \mathrm{H}_{12} \mathrm{~N}_{2} \mathrm{O}_{5}$ (336) calcd. (\%) C $64.28 ; \mathrm{H} 3.57 ; \mathrm{N} 8.33$.

4-(5'-Formyl-1-(4"'-chlorophenyl)-1H-pyrrol-2-yl) benzoic acid (25): Brown crystals, m.p. $250{ }^{\circ} \mathrm{C}(\mathrm{EtOH})$ FTIR $\left(\mathrm{KBr}, v_{\max }, \mathrm{cm}^{-1}\right): 2348.57$ (aromatic ring) $1676.17(\mathrm{C}=\mathrm{O}$ aldehyde) 1045.88 (C-Cl bond), 2979.83 (-OH acid), ${ }^{1} \mathrm{H}$ NMR spectrum $\left(\mathrm{CDCl}_{3}, 300 \mathrm{MHz}\right), \delta \mathrm{ppm}(\mathrm{J}, \mathrm{Hz}): 9.683(\mathrm{~s}, 1 \mathrm{H}$, CHO), 8.301-7.331 (m, 8H, Ar-H), 6.960 (d, 1H, pyrrole-H, $J$ $=3.50 \mathrm{~Hz}), 6.934(\mathrm{~d}, 1 \mathrm{H}$, pyrrole- $\mathrm{H}, J=3.50 \mathrm{~Hz}), 11.945(\mathrm{~s}$, $\mathrm{COOH}){ }^{13} \mathrm{C} \mathrm{NMR}$ spectrum $\left(\mathrm{CDCl}_{3}, 75 \mathrm{MHz}\right) \delta$ ppm: 178.79 ( $\mathrm{C}=\mathrm{O}$ aldehyde), 162.56, 147.95, 142.62, 132.86, 130.82, $130.26,130.22,125.07,122.92,118.76,110.90$ (Ar-C) Mass spectrum (EI, $70 \mathrm{eV},\left(\mathrm{I}_{\text {rel }} \%\right): m / z 371\left[\mathrm{M}^{+}+2\right], 325\left[\mathrm{M}^{+}\right]$ (4), $216\left[\mathrm{M}^{+}-\mathrm{PhCOOH}\right]$ (100) found (\%) C 66.24; H 3.78; N $4.11 \mathrm{C}_{18} \mathrm{H}_{12} \mathrm{ClNO}_{3}$ (325) calcd. (\%) C 66.46; H 3.69; N 4.30.

4-(5'-Formyl-1-(4"'-bromophenyl)-1H-pyrrol-2-yl) benzoic acid (26): Brown crystals, m.p. $262^{\circ} \mathrm{C}(\mathrm{EtOH})$ FTIR (KBr, $\left.v_{\max }, \mathrm{cm}^{-1}\right): 2983.52(-\mathrm{OH}) 1609.50(\mathrm{C}=\mathrm{O}$ aldehyde), $1673.98(\mathrm{C}=\mathrm{O}$ carboxylic acid) 2363.93 (aromatic ring), 1045.95 (C-Br bond), $2829.98 \mathrm{~cm}^{-1}$ (C-H stretch of aldehyde), ${ }^{1} \mathrm{H}$ NMR spectrum $\left(\mathrm{CDCl}_{3}, 300 \mathrm{MHz}\right), \delta \mathrm{ppm}(\mathrm{J}, \mathrm{Hz}): 9.780(\mathrm{~s}$, 1H, CHO), 8.406-7.569 (m, 8H, Ar-H), 6.965 (d, 1H, pyrrole$\mathrm{H}, J=2.95 \mathrm{~Hz}), 6.732$ (d, 1H, pyrrole-H, $J=2.95 \mathrm{~Hz}), 11.675$ $(\mathrm{s}, \mathrm{COOH}){ }^{13} \mathrm{C} \mathrm{NMR}$ spectrum $\left(\mathrm{CDCl}_{3}, 75 \mathrm{MHz}\right) \delta \mathrm{ppm}$ : 178.45 ( $\mathrm{C}=\mathrm{O}$ aldehyde), $165.67(\mathrm{COOH}), 141.67,130.22$, 125.03, 123.11, 122.09, 109.30 (Ar-C) mass spectrum (EI, 70 $\mathrm{eV},\left(\mathrm{I}_{\mathrm{rel}} \%\right): \mathrm{m} / z 371\left[\mathrm{M}^{+}+2\right], 369\left[\mathrm{M}^{+}\right](12), 216\left[\mathrm{M}^{+}-\mathrm{PhCOOH}\right]$ (50) found (\%) C 58.18; H 3.39; N $3.61 \mathrm{C}_{18} \mathrm{H}_{12} \mathrm{BrNO}_{3}$ (369) calcd. (\%) C 58.53; H 3.25; N 3.79 .

4-(5'-Formyl-1-(4"-carboxyphenyl)-1H-pyrrol-2-yl) benzoic acid (27): Light yellow crystals, m.p. $202^{\circ} \mathrm{C}(\mathrm{EtOH})$ FTIR $\left(\mathrm{KBr}, v_{\max }, \mathrm{cm}^{-1}\right): 2341.42$ (aromatic ring) $1680.53(\mathrm{C}=\mathrm{O}$ acid), 1593.20 (C=O aldehyde) 2980.73 (-OH acid), ), ${ }^{1} \mathrm{H}$ NMR spectrum $\left(\mathrm{CDCl}_{3}, 300 \mathrm{MHz}\right), \delta \mathrm{ppm}(\mathrm{J}, \mathrm{Hz}): 9.636(\mathrm{~s}, 1 \mathrm{H}$, CHO), 8.314-7.425 (m, 8H, Ar-H), 6.812 (d, 1H, pyrrole-H, $J=5.50 \mathrm{~Hz}), 6.742(\mathrm{~d}, 1 \mathrm{H}$, pyrrole- $\mathrm{H}, J=3.50 \mathrm{~Hz}), 11.410(\mathrm{~s}$, $\mathrm{COOH}){ }^{13} \mathrm{C} \mathrm{NMR}$ spectrum $\left(\mathrm{CDCl}_{3}, 75 \mathrm{MHz}\right) \delta$ ppm: 179.85 $(\mathrm{C}=\mathrm{O}$ aldehyde $), 166.39(\mathrm{COOH}), 148.76,142.35,133.78$, $132.89,131.09,129.65,123.64,122.70,119.62,110.69(\mathrm{Ar}-$ C) mass spectrum (EI, $70 \mathrm{eV},\left(\mathrm{I}_{\text {rel }} \%\right): m / z, 336\left[\mathrm{M}^{+}\right](4), 304$ $\left[\mathrm{M}^{+}-\mathrm{CHO}\right](50)$ found (\%) C 68.01; H 3.72; N $4.11 \mathrm{C}_{19} \mathrm{H}_{13} \mathrm{NO}_{5}$ (335) calcd. (\%) C 68.05; H 3.88; N 4.17.

\section{RESULTS AND DISCUSSION}

Various arylfurfurals (4-7) were prepared in 40-70\% yield by catalytic arylation of furfural with arenediazonium salts according to Meerwein method ${ }^{29}$. These were treated with 
appropriate anilines at reflux in ethanol in the presence of a catalytic amount of hydrochloric acid to provide, in good yields, the respective 1,5-diarylpyrrole-2-carbaldehydes (8-27) by a ring transformation reaction (Scheme-I). The procedure followed the one reported for the preparation of 1-arylpyrrole2-carbaldehydes from furfural and anilines ${ }^{27,28}$. Lewis and Mulquiney ${ }^{30-32}$ have extensively studied the reaction of aryl amines and furan-2-carbaldehyde and have identified the formation of the intermediate "Stenhouse salt" (Scheme-II).
Which under the acid or basic conditions leads to the formation of various products: 1-aryl-3-hydroxypyridine and 1-arylpyrrole2-carbaldehydes.

FTIR analysis: Assignment of selected characteristics IR bands provides significant indication for the formation of diaryl pyrrole-2-carbaldehydes. The aldehydic group $(\mathrm{C}=\mathrm{O})$ and $(\mathrm{C}-\mathrm{H})$ absorbed in the expected region; $(\mathrm{C}=\mathrm{O})$ in the 1610-1681 $\mathrm{cm}^{-1},(\mathrm{C}-\mathrm{H})$ stretch of aldehydes show a weak band around 2846-2863 $\mathrm{cm}^{-1}$, while $\mathrm{NO}_{2}$ group present in some compounds<smiles>[R]c1ccc(-c2ccc(C=O)o2)cc1</smiles>

4-7<smiles>[R]c1ccc(-c2ccc(C=O)o2)cc1</smiles>

$\mathrm{R}=\mathrm{NO}_{2}, \mathrm{Cl}, \mathrm{Br}, \mathrm{COOH} \mathrm{R}^{\prime}=\mathrm{H}, \mathrm{NO}_{2}, \mathrm{Cl}, \mathrm{Br}, \mathrm{COOH}$

Scheme-I

Mechanism:

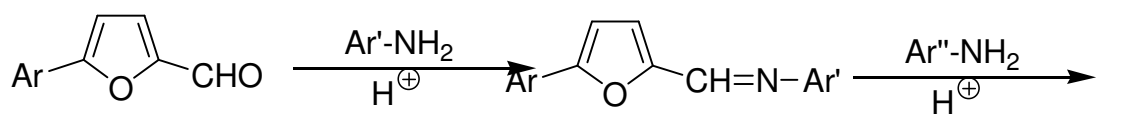

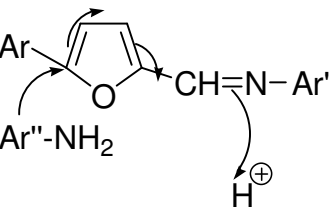

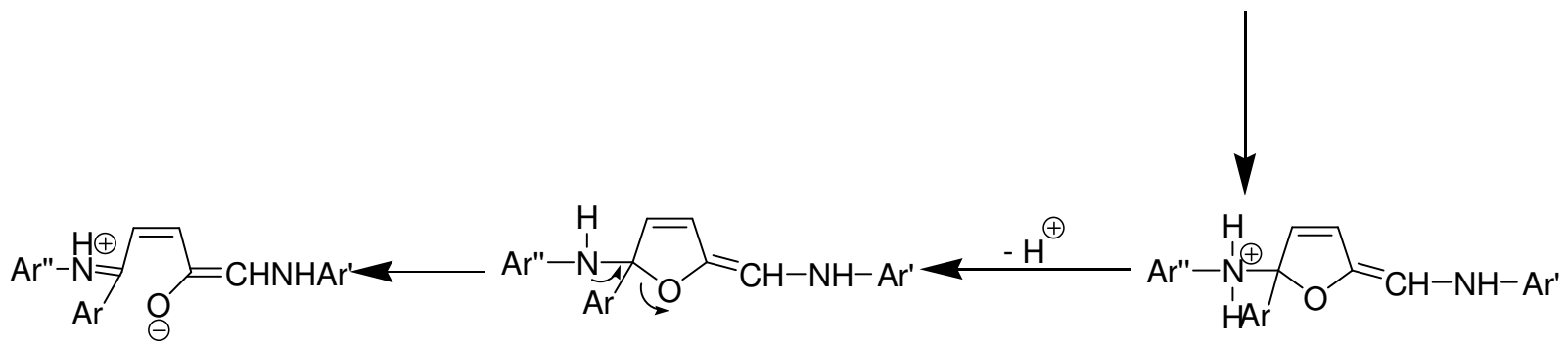

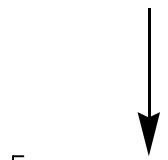

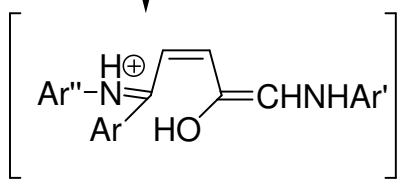

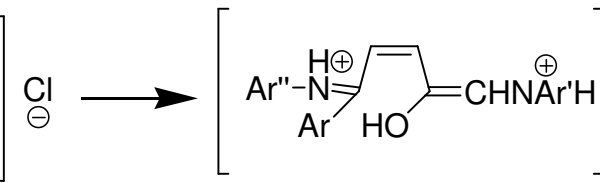<smiles>CC(Cl)Cl</smiles>

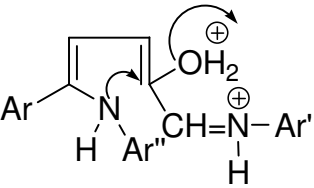<smiles>Cc1ccc(Br)n1[Al]</smiles>

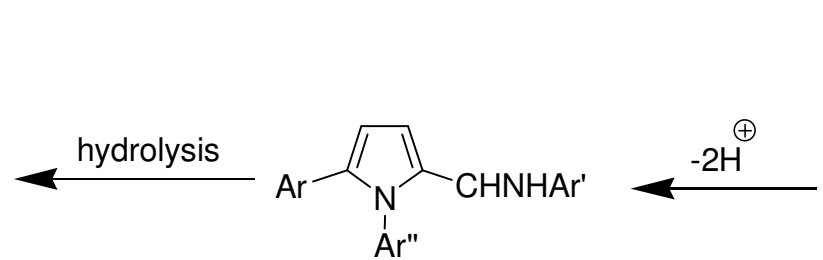

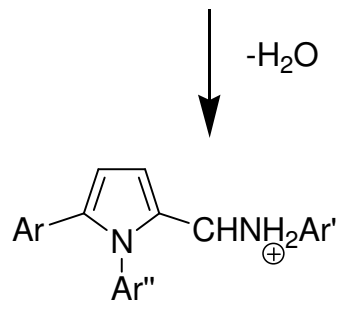

Scheme-II 
shows characteristics strong band in 1341-1298 and at 1600$1584 \mathrm{~cm}^{-1}$ region.

${ }^{1} \mathrm{H}$ NMR analysis: The ${ }^{1} \mathrm{H}$ NMR spectra $\left(300 \mathrm{MHz}, \mathrm{CDCl}_{3}\right)$ of the starting aryl furfurals and diaryl pyrrole-2-aldehydes show characteristic signals between 9.64-9.72 ppm for aldehydic proton and two doublet at 6.78-6.98 and 7.29-7.96 ppm for the pyrrole ring hydrogens.

${ }^{13} \mathrm{C}$ NMR analysis: Finally, ${ }^{13} \mathrm{C}$ NMR $\left(75 \mathrm{MHz}, \mathrm{CDCl}_{3}\right)$ spectra of all compounds were recorded and spectral signals are in good agreement with the structures. Carbon of $\mathrm{C}=\mathrm{O}$ displayed signals at 177.21-177.72 ppm in the arylfuran-2carbaldehydes and diarylpyrrole-2-carbaldehydes while pyrrole ring carbons show signals for their characteristic positions.

Mass spectra: Mass spectra of all compounds were recorded and their values are given in the experimental section. These characterize the formation of starting arylfuran-2carbaldehydes and diarylpyrrole-2-carbaldehydes.All the new compounds displayed expected molecular ion peaks.

\section{Conclusion}

A good and efficient method for the preparation of diarylpyrrole-2-carbaldehydes by the ring transformation of arylfuran-2-carbaldehydes in acidic media is presented in this paper. From these diaryl pyrrole-2-carbaldehydes, various other heterocyclic compounds can be synthesized. Further work is in progress.

\section{ACKNOWLEDGEMENTS}

The authors (SA and AN) acknowledged the financial support by HEC, Govt. of Pakistan in the form of an indigenous $\mathrm{PhD}$ fellowship.

\section{REFERENCES}

1. J. Lehuedu, B. Fauconneau, L. Barrier, M. Ourakow, A. Piriou and J.M. Vierfond, Eur. J. Med. Chem., 34, 991 (1999).

2. M.V. Raimondi, S. Cascioferro, D. Schillaci and S. Petruso, Eur. J. Med. Chem., 41, 1439 (2006).

3. L.C.L. Cara, M.E. Camacho, M.D. Carrión, V. Tapias, M.A. Gallo, G Escames, D. Acuña-Castroviejo, A. Espinosa, A. Entrena, Eur. J. Med. Chem., 44, 2655 (2009).

4. K. Abu-Rabeah and R.S. Marks, Sens. Actuators B, 136, 516 (2009).

5. J.H. Liu, Q.C. Yang, T.C.W. Mak and H.N.C. Wong, J. Org. Chem., 65, 3587 (2000).

6. H. Tao, I. Hwang and D.L. Boger, Bioorg. Med. Chem. Lett., 14, 5979 (2004).

7. R.K. Dieter and H. Yu, Org. Lett., 2, 2283 (2000)
8. N. Iwasawa, K. Maeyama and M. Saitou, J. Am. Chem. Soc., 119, 1486 (1997).

9. A. Furstner, H. Weintritt and A. Hupperts, J. Org. Chem., 60, 6637 (1995).

10. A. Katritzky, J. Jiang and P.J. Steel, J. Org. Chem., 59, 4551 (1994).

11. M. Periasamy, G. Srinivas and P. Bharati, J. Org. Chem., 64, 4204 (1999).

12. C. Paal, Chem. Ber., 17, 2557 (1884); L. Knorr, Chem. Ber., 17, 2863 (1884); J. Chen, H. Wu, Z. Zheng, C. Jin, X. Zhang and W. Su, Tetrahedron Lett., 47, 5383 (2006)

13. A. Zarghi and S. Arfaei, Iran. J. Pharm. Res., 10, 655 (2011).

14. G. Dannhardt and W. Kiefer, Eur. J. Med. Chem., 36, 109 (2011).

15. G. Gaetano, M.B. Donati and C. Cerletti, Trends Pharmacol. Sci., 24, 245 (2003)

16. G.A. Fitzgerald, N. Engl. J. Med., 351, 1709 (2004).

17. W.W. Wilkerson, W. Galbraith, K. Gans-Brangs, M. Grubb, W.E. Hews, B. Jaffee, J. P. Kenney, J. Kerr and N. Wong, J. Med. Chem., 37, 988 (1994).

18. M. Biava, G.C. Porretta A. Capelli, S. Vomero, F. Manetti, M. Botta, L. Sautebin, A.D. Rossi, F. Makovec and M. Azini, J. Med. Chem., 48, 3428 (2005).

19. M. Rovini, A. Capelli, S. Vomero, F. Manetti, M. Botta, L. Sautebin, A.D. Rossi, C. Pergola, C. Ghelardini, M. Norcini, A. Giordani, F. Makovec, P. Anzelloti, P. Patrignani, M. Biava and M. Anzini, J. Med. Chem., 51, 4476 (2008).

20. M. Biava, G.C. Porretta, G. Poce, C. Battilocchio, F. Manetti, M. Botta, S. Forli, L. Sautebin, A. D. Rossi, C. Pergola, C. Ghelardini, N. Galeotti, F. Makovec, A. Giordani, P. Anzelloti, P. Patrignani and M. Anzini, J. Med. Chem., 53, 723 (2010).

21. M. Biava, G.C. Porretta, G. Poce, A.D. Logu, M. Saddi, R. Meleddu, F. Manetti, F.D. Rossi and M. Botta, J. Med. Chem., 51, 3644 (2008).

22. M. Biava, G.C. Porretta, G. Poce, A.D. Logu, R. Meleddu, F.D. Rossi, F. Manetti and M. Botta, Eur. J. Med. Chem., 44, 4734 (2009).

23. A. Hall, S.H. Brown, P.I. Chessell, A. Chowdhury, N.M. Clayton, T. Coleman, G.M.P. Giblin, B. Hammond, M.P. Healy, M.R. Johnson.A. Metcalf, A.D. Michel, A. Naylor, R. Novelli, D.P. Spalding, J. Sweeting, Biorg. Med. Chem. Lett., 17, 732 (2007).

24. A. Hall, S.H. Brown, C. Budd, N.M. Clayton, G.M.P. Giblin, P. Goldsmith, T.G. Hayhow, D.N. Hurst, A. Naylor, D.A. Rawlings, T. Scoccitti, A.W. Wilson and W.J. Winchester, Bioorg. Med. Chem. Lett., 19, 497 (2009).

25. J. Sanchez and M.D. Pujol, Tetrahedron, 55, 5593 (1999).

26. D. Bandyopadhyay, S. Mukherjee and B.K. Banik, Molecules, 15, 2520 (2010).

27. S.I. Burmistrov and V.M. Sannikova, Voprosy Khimii I Khimicheskoi Tekhnologii, 20, 54 (1979); Chem. Abstr., 92, 180923 (1980).

28. E. Baum, T.E. Goldovskaya, V.G. Kul'nevich and O.V. Maiorova, Khim. Getrot. Soedin, 1062 (1981); Chem. Abstr., 95, 203666 (1981).

29. J. March, Advanced Organic Chemistry: Reactions, Mechanism and Structure, Wiley Interscience, New York, edn. 4, pp. 712-715 (1992).

30. K.G. Lewis and C.E. Mulquiney, Aust. J. Chem., 30, 463 (1977).

31. K.G. Lewis and C.E. Mulquiney, Aust. J. Chem., 32, 1079 (1979).

32. G.E. Lewis and D.L. Lill, Aust. J. Chem., 38, 953 (1985).

33. B.S. Holla, P.M. Akberali and M.K. Shivananda, IL Farmaco, 56, 919 (2001). 